\title{
A Real Time Vision based Smart Bulb using Image Processing
}

\author{
Dashrath Mahto \\ M.Tech. Scholar \\ Oriental Institute of Science \& Technology, Bhopal
}

\author{
Sreeja Nair \\ Associate Professor \\ Oriental Institute of Science \& Technology, Bhopal
}

\begin{abstract}
In recent years, light bulb is getting smarter because of its smart behavior. It means that it can be controlled via smartphone through a wireless connection and even you may control it when you are not at your home. Now a day's market place has been occupied with the smart bulbs, replacing CFLs and traditional LED. It is totally differs from the traditional bulb even much more expensive as compare to the traditional one. Smart bulb offers remote control feature along with schedule timer, it means that you have a smartphone as a remote and you can also set a timer to turn off or on these bulbs. Even it can be controlled through GPS installed on your smartphone. It means that when you are a bit far away from your home and you forget to turn off your bulbs, then you can turn off it, your bulb is almost another smartphone that is why it is expensive as compared to the traditional bulb. Now it's time to replace this smart bulb from new era with much smarter bulb which is going to be proposed, i.e. Real Time Vision Based Smart Bulb Using Image Processing. It will work like a human vision, a bulb which does not require any manual control, it will be operated automatically. It means that it will automatically sense when it should be off and on. When no one is present in the room, then it will automatically off and when you come, it will automatically on. One more feature is very effective in it, is when you present in the room and there is no movement for half an hour, then it will automatically off, because it is practically demonstrated that no one can be totally stable for half an hour when he is not sleeping or when he is conscious. He must have any kind of movement if he is conscious. He must be sleeping if he is not responding for half an hour. It will consider that you are sleeping and bulb should be turned off. Even you may turn off or on this feature as per your desire. Definitely it will save your electricity when you are not in your home or even you are at home. It is intelligent enough to control the system by itself and no smartphone or any remote control device is required. It will be the most intelligent light bulb till now.
\end{abstract}

\section{Keywords}

Smart Bulb, Image Processing, CFL, LED, MATLAB, GPS etc.

\section{INTRODUCTION}

\subsection{Introduction of Present System}

Today's light bulb control through switches, it will require man power to turn off or on. Actually LED was introduced to replace CFLs because of power consumption. LED requires much lesser power as compare to CFLs. But later this LED technique gets enhanced with wireless control system. It means that there will be a Wi-Fi device embedded in the bulb and it can be connected to your smartphone via application and with the help of this you may control your bulbs as per your comfort. Even you may control your bulb when you will not be in your house, it will be controlled through GPS which is installed in the bulb as well as in your smartphone.

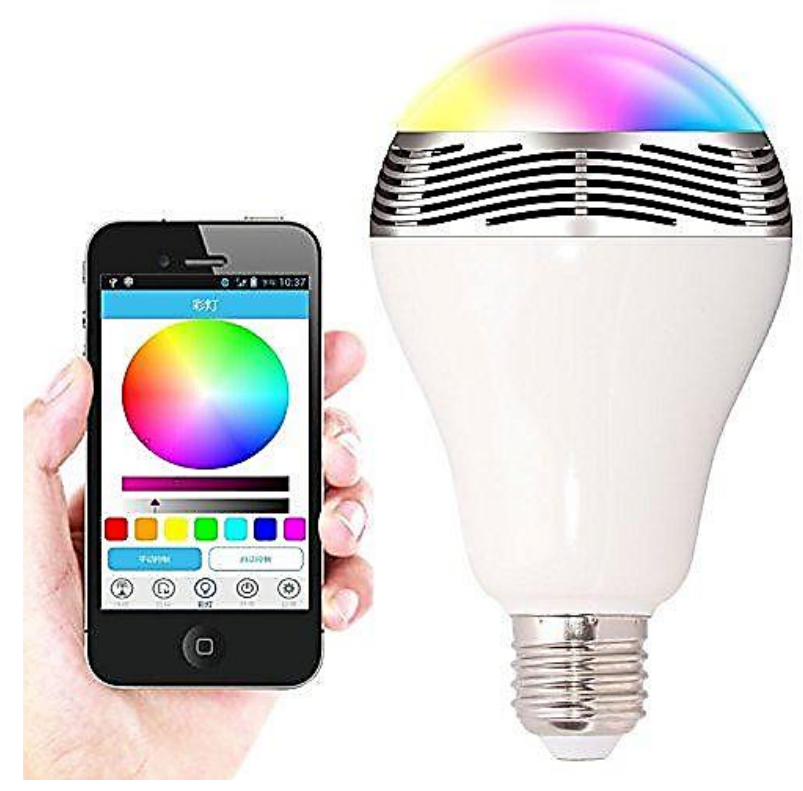

Fig. 1.1.1 Smart LED Bulb

Present smart LED can also emit light in various colors, it has color effect features. There are so many kinds of smart LED bulbs available in the market such as Philips Hue Connected Bulb, Philips Hue White, C by GE Starter Pack, Cree Connected LED Bulb, GE Link Connected LED, LIFX Color 1000, Philips Hue Wireless Dimming Kit, TikTeck Smart LED Light Bulb, MiPow Playbulb Rainbow, Stack Down light Starter Kit and many more. Few of them can sense low light and able to turn it brighter in that case and a few of them can emit multiple colors of light and rest all are able to connect to smartphone for remote control.

\subsection{Introduction of Proposed System}

\subsubsection{Smart Light Bulb}

To replace present system, we have new promising concept which is intelligent enough for real time decision making. We proposed a new promising system which will be able to monitor real time activity. It is slightly differ from the present system, here we are not going to control it through your smartphone and we are going to develop a system which will be intelligent enough to take decisions on real time with practical sense i.e. when it should be turned off or on. Suppose you are working on living room and after some time you go to your bed room to sleep, but you forgot to turned off your bulb, in this case if we are using a traditional bulb then it will remain on until you turned it off and if we are using smart 
LED then it will also remain on because you will also have to turn off it manually through your smartphone, but if we talk about our system then it will check if you are not present in your living room then it will automatically getting off. No need to turn it off manually, even when you come back to your living room it will 'on' your bulb automatically. One more thing which is not possible for traditional bulb as well as present smart LED is that when you are working in your living room and after a while, you got sleep there, while working then if we talk about traditional system, the light bulb will remain on till your effort will not come, it is as similar with present smart LED but our system has a sense if you are stable for 30 minutes or no movement with your body, definitely you are sleeping as well, because it is practically demonstrated that if someone has no movement till 30 minutes then definitely he is not conscious. In this case our system will turn off the switch automatically and it will turn on when you show your palm as a signal. It is totally incredible to observe and it will make your lifestyle easier.

\section{PREVIOUS WORKS}

\subsection{Review on existing systems:}

There are so many papers proposed for smart LED but all these systems lacking somewhere. Few of them are manually controlled and few of them can control the light bulb, automatic, but why they are not so much efficient let it to be more precise.

Smart LED lighting system implementation using Human tracking US/IR sensor proposed by Daeho Kim1, Junghoon Lee1 and Yeongmin Jang2, Jaesang Cha1*in 978-1-45771268-5/11/\$26.00 @2011 IEEE. [1]

This paper was proposed in 2011 which was based on ultrasonic sensor (US) and infrared sensor (IR) to track human.

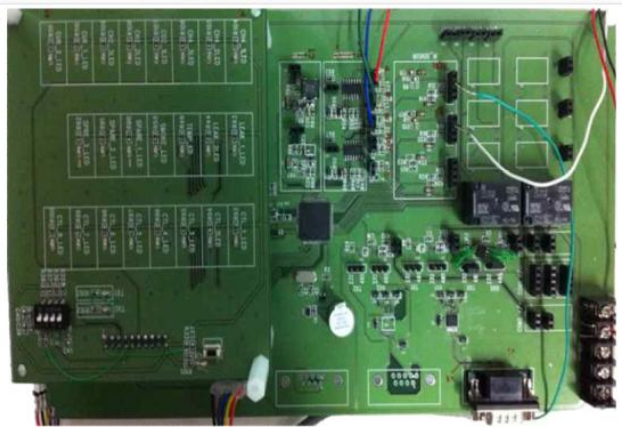

Fig. 1.2.1.1 The implemented MCU board

By tracking humans, it operates the bulb automatically, but these sensors are not enough intelligent to recognize human, these sensors are also very expensive to embedded. That is why there is no practical model proposed in the market based on these sensors.

Smart Personal Sensor Network Control for Energy Saving in DC Grid Powered LED Lighting System proposed by Yen Kheng Tan in 2012 on IEEE. [2]

This paper was proposed in 2012 which focused on saving energy efficiently using low power DC grid instead of AC power. It does not have an automatic control system, but it has a feature of controlling energy automatically. It has ability to perform similar lighting, even in low voltage because it is using DC power which is to be converted from AC power to perform efficiently. But there is no sensing capability to turn off or on the switches automatically.

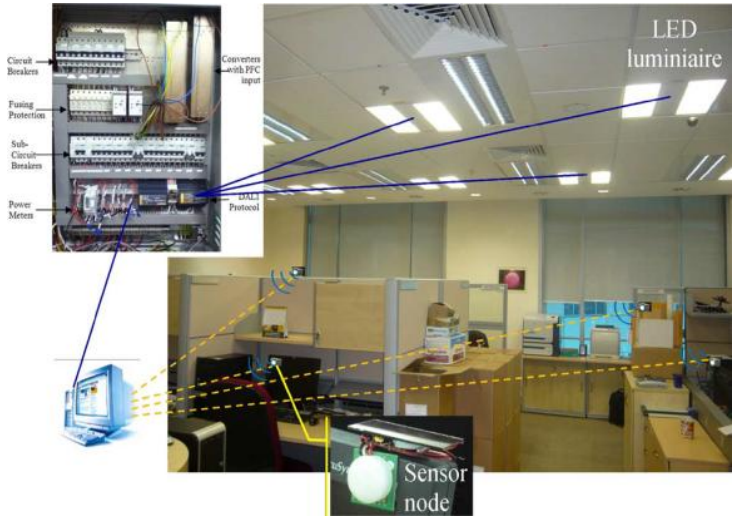

Fig. 1.2.1.2 Deployment of smart personal sensor network control for energy saving in DC grid powered LED lighting system

Development of Smart LED Lighting System Using MultiSensor Module and Bluetooth Low Energy Technology proposed by Young Seek Cho,Jaerock Kwon, Seyeong Choi and Dae-Hee Park in 2014 IEEE SECON Posters IEEE International Conference on Sensing, Communications and Networking (SECON). [3]

This paper was proposed in 2014 which developed a smart bulb which can be controlled through a smartphone using Bluetooth, it means that it will communicate via Bluetooth to control the function of the bulb. It has so many sensors like temperature measuring sensor, light intensity sensor and much more. But this system depends on your instructions. This system does not operate automatically. Bluetooth is not a strong medium as compare to Wi-Fi or others.

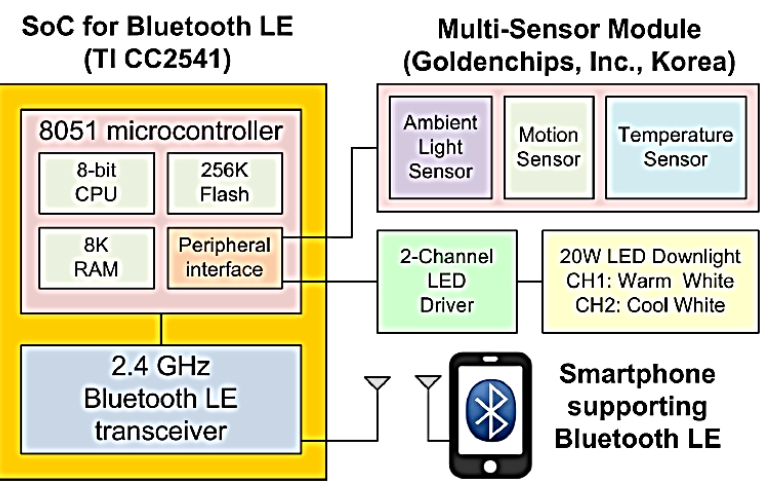

Fig. 1.2.1.3 High level block diagram of the proposed LED lighting system

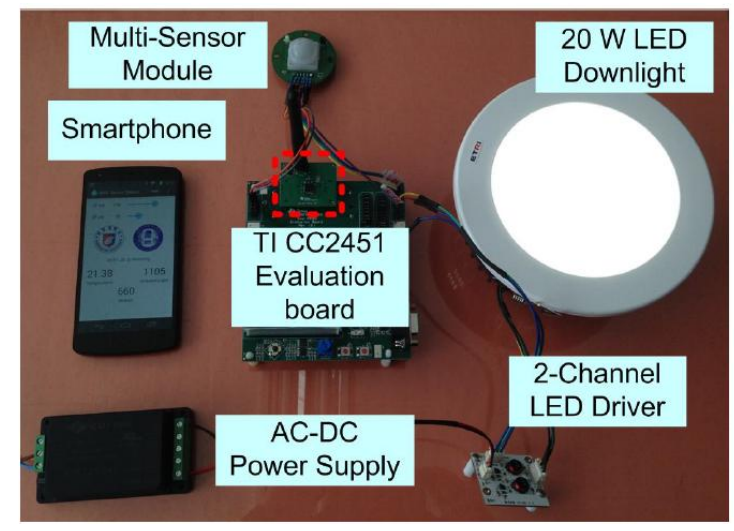

Fig. 1.2.1.4 A picture of the smart LED lighting system implemented 
Even there so many companies like Philips and many more proposed a smart bulb which can be controlled through your smartphone using Wi-Fi and GPS. As per their implementation closure they didn't publish their idea of forming smart bulb.

\section{PROBLEM STATEMENTS}

The existing system is not intelligent enough to sense when it should turn off or on. It is manually controlled through your smartphone, it has the convenience of remote access, but when you are sleeping or when you are not at your house, you have to check whether it is on or not, then you may turn off or on it, some kind of effort and concern are required by this system. Suppose if you are sleeping and you forgot to 'off' your bulb then how you will turn it off while sleeping, you will have to awake and turn it off manually either by your smartphone or switch. One more thing is that if you go out from your house and you forgot to 'off' you bulb and suddenly you remind that you forgot something and you check it via your smartphone and you turned it off, is it liable? In this busy world you do not remember anything, you don't have time to check whether you turned off your bulbs or not, if you remember to check it through your smartphone then why not while leaving your home. So, by observing all this we require a totally self-decision making bulb or much smarter bulb which reduces human efforts in all aspects. And present system is not that much more intelligent that is why this system is lacking somewhere which is required to overcome.

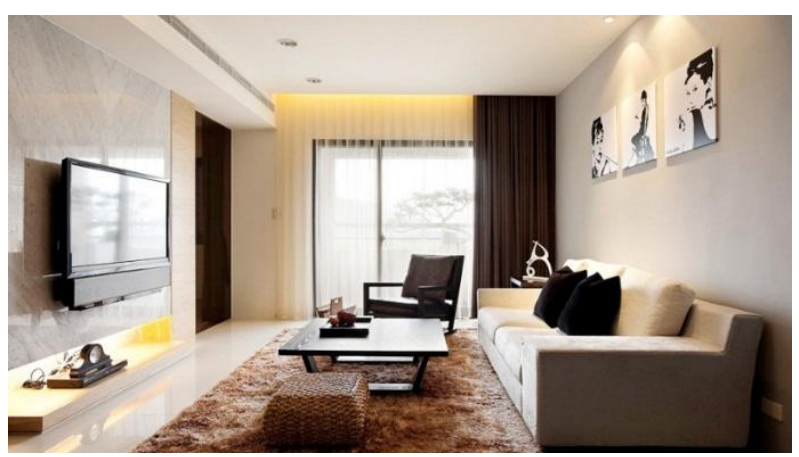

Fig. 3.1 Target Place

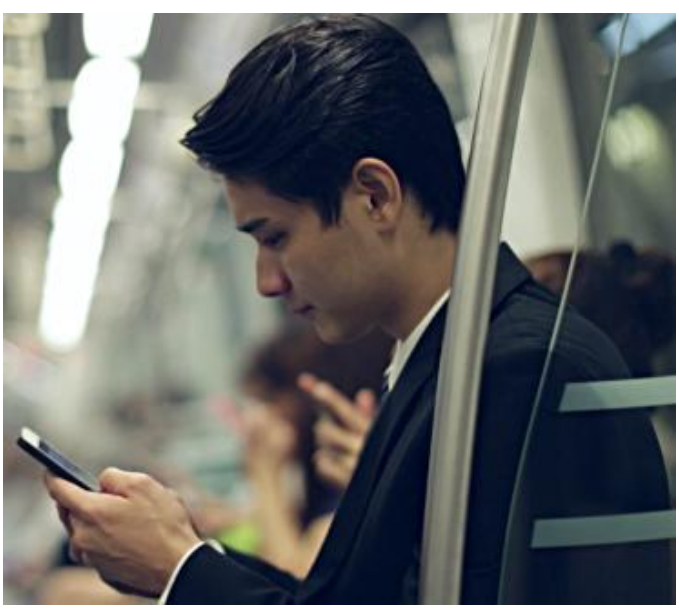

Fig. 3.2 Control via Smartphone

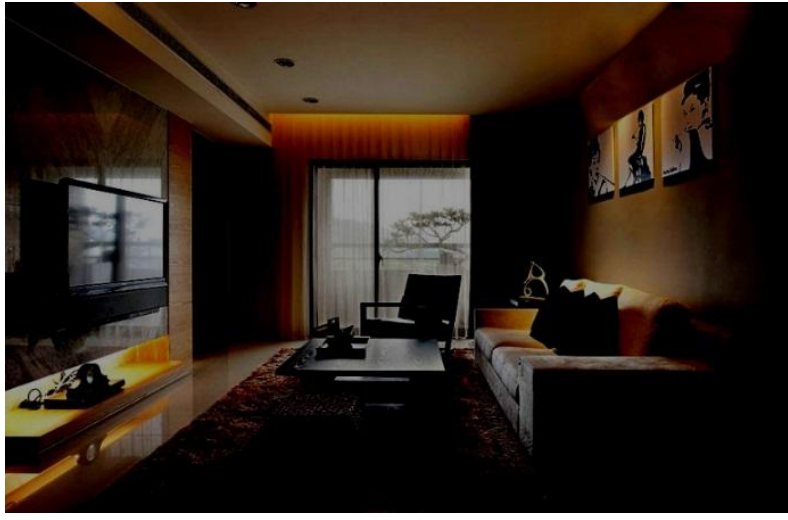

Fig. 3.3 Bulb Controlled via Smartphone

\section{PROPOSED WORK}

\subsection{Smart Light Bulb}

In this system, there will be a camera which will be able to recognize changes on the basis of your triggered background image and works automatically. It means that a light integrated with a camera which is able to sense if there is a person in a room or not and it is also able to sense your activity whether you are sleeping or not and accordingly it turn off or on. Suppose you are working with your laptop in your living room as figure 4.1.1 given below.

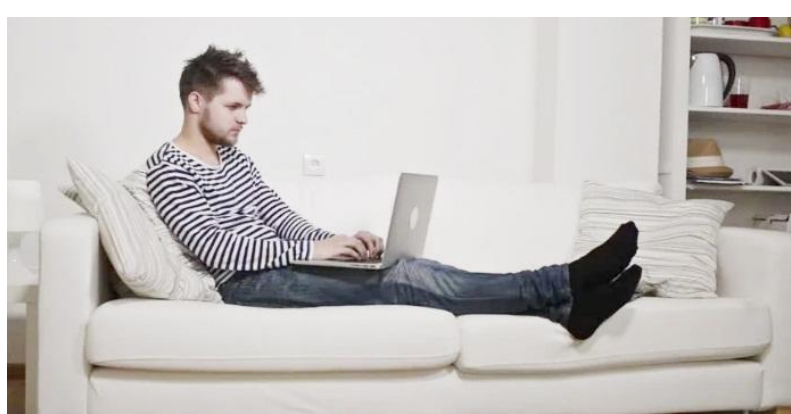

Fig. 4.1.1 Working on Living Room

Suddenly you got sleep, then by present smart LED it is impossible to turn off light automatically, you will have to turn it off either by your smartphone or your switch.

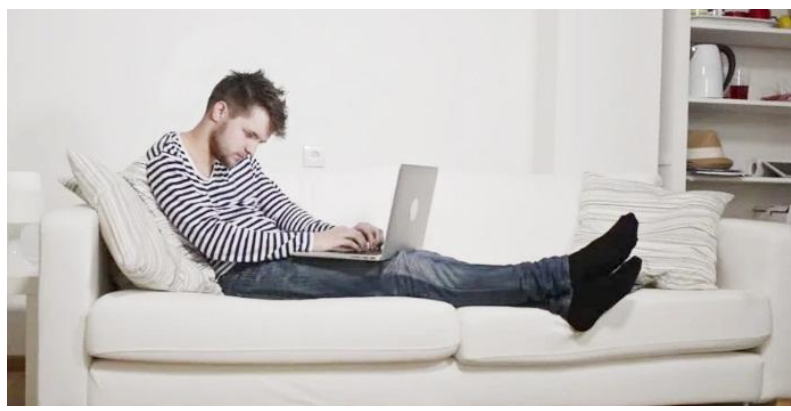

Fig. 4.1.2 Sleep Detection

But the proposed system will sense your movement and accordingly it will turn off your bulb automatically as figure 4.1.3 given below. 


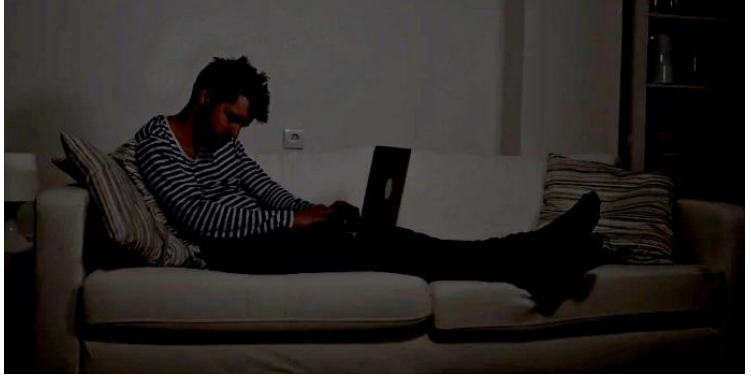

Fig. 4.1.3 Action after sleep detection

One more feature is that, suppose you are working in your living room or somewhere else and after a while you went to sleep or you went somewhere else, then in this case tradition bulb will remain turned on till you do not switch it off and if it is smart LED then you have to turn it off by your smartphone, but if you are sleeping then how you will switch it off or if you are not in your home and you forgot to check via your smartphone then it does not turn off automatically. But proposed system has ability to sense if you are not in your room then it will turn off your bulb automatically, then whether you are sleeping in your bedroom or you are not in your home or you forgot to switch off your bulb. Suppose a person is working in his living room as shown in the figure 4.1.4 given below and after a while he went to sleep in his bedroom and he forgot to 'off' bulb then system is able to sense whether is there someone present in the room or not

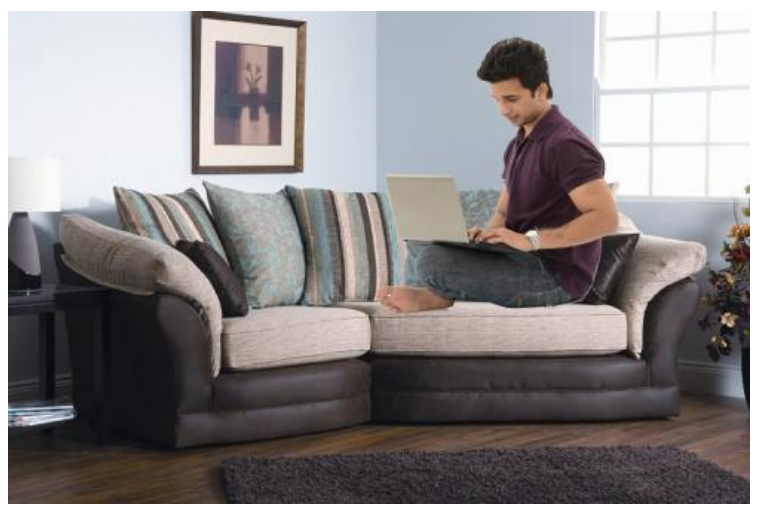

Fig. 4.1.4 Working on the living room

If the system detects no one is present in the room as we can see in the figure 4.1.5 no one is present in that room, so in that case it will turn off the bulb automatically as we can see in figure 4.1.6 where the bulb is turned off automatically because no one is there and whenever you will come to your living room it will turn on.

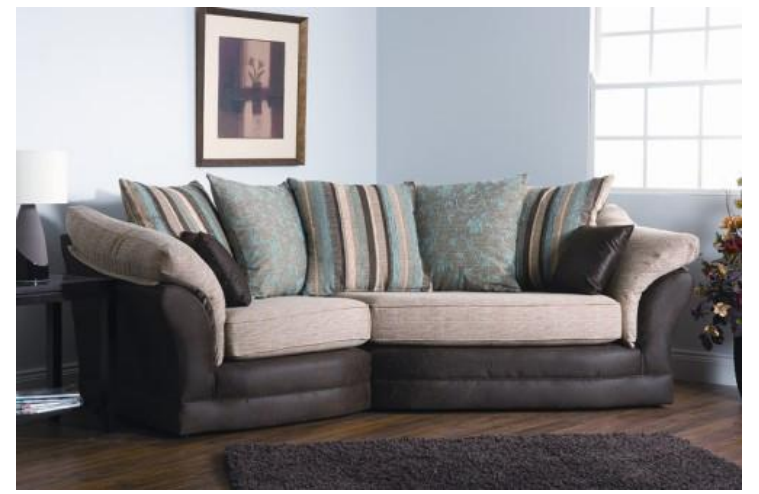

Fig. 4.1.5 No one is detected in the room

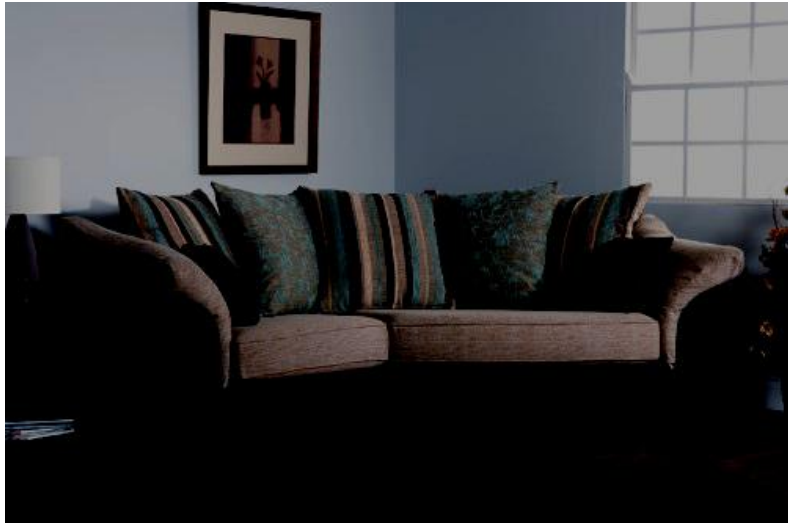

Fig. 4.1.6 Action after detection

\section{PROPOSED METHODOLOGY}

An image is defined by the mathematical function $f(x, y)$ where $\mathrm{x}$ and $\mathrm{y}$ are the two coordinates horizontally and vertically. The value of $\mathrm{f}(\mathrm{x}, \mathrm{y})$ at any point gives the pixel value at that point of an image. This image here is a $2 \mathrm{D}$ array of numbers ranging between 0 and 255 .

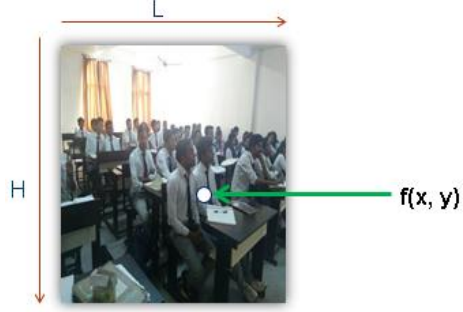

$$
\begin{aligned}
& \text { Here: } \\
& 0 \leq x \leq H \quad \& \quad 0 \leq y \leq L \\
& L=\text { length of image } \\
& H=\text { height of image } \\
& f(x, y)=\text { image intensity or } \\
& \text { color of particular point. } \\
& \text { Imin } \leq f(x, y) \leq I m a x
\end{aligned}
$$

Fig. 5.1.1 $\mathrm{X}$ and $\mathrm{Y}$ axis

Representation of Digital Images: An image can be represented in the form of a finite 2D matrix. Each matrix element represented by one of the set of discrete values i.e. intensity value.

$$
\left[\begin{array}{ccc}
f(0,0) & f(0,1) & f(0,2) \ldots \ldots \ldots . f(0, N-1) \\
f(1,0) & f(1,1) & f(1,2) \ldots \ldots \ldots \ldots f(1, N-1) \\
f(2,0) & f(2,1) & f(2,2) \ldots \ldots \ldots \ldots f(2, N-1) \\
: & &
\end{array}\right] \quad \begin{aligned}
& \text { Image Intensity: refers to } \\
& \text { the amount of light or the } \\
& \text { numerical value of pixel. }
\end{aligned}
$$

Fig. 5.1.2 Matrix Representation

Digital Image processing is a method to convert an image into digital form and perform some operations on it, in order to get an enhanced image or to extract some useful information from it. Here is the $8 \times 8$ pixel grid on 256 pixel image.

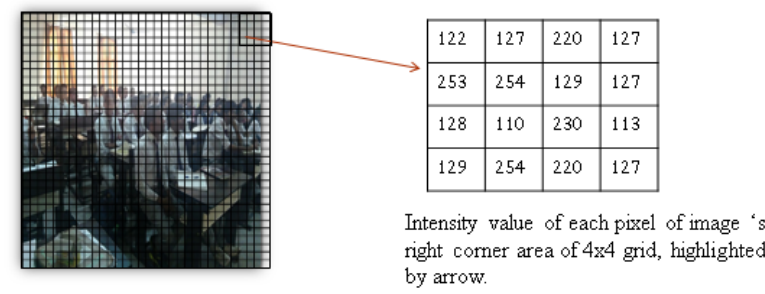

Fig. 5.1.3 Digital Image Processing 
Histogram: The histogram of an image normally refers to a histogram of the pixel intensity values. This histogram is a graph showing the number of pixels in an image at each different intensity value. For an 8-bit grayscale image there are 256 different possible intensities, and so the histogram will graphically display 256 numbers showing the distribution of pixels amongst those grayscale values.

The histogram of a digital image with gray levels in the range $[0, L-1]$ is a discrete function $h\left(r_{k}\right)=n_{k}$. Where $r_{k}$ is the kth gray level and $n_{k}$ is the number of pixels in the image having gray level $r_{k}$, the total number of pixels in the image denoted by $n$.

Normalized Histogram: A normalized histogram is given by $\mathrm{p}\left(\mathrm{r}_{\mathrm{k}}\right)=\mathrm{n}_{\mathrm{k}} / \mathrm{n}$, for $\mathrm{k}=0,1, \ldots \mathrm{L}-1 . \mathrm{p}\left(\mathrm{r}_{\mathrm{k}}\right)$ gives an estimate of the probability of occurrence of gray level $r_{k}$.
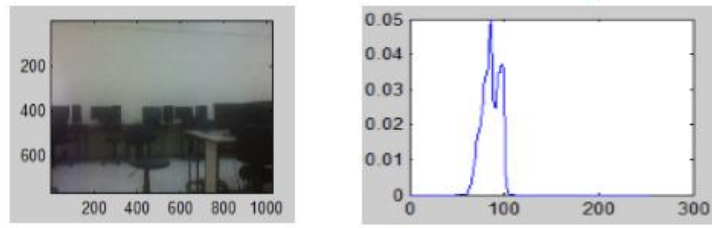

Fig. 5.1.4 Histogram of an Image

The horizontal axis of each histogram plot corresponds to gray level values, $\mathrm{r}_{\mathrm{k}}$. The vertical axis corresponds to values of $h\left(r_{k}\right)=n_{k}$ or $p\left(r_{k}\right)=n_{k} / n$, if the values are normalized.

Histogram Equalization: Histogram equalization is a technique for adjusting image intensities to enhance contrast. Let $\mathrm{f}$ be a given image represented as a $\mathrm{mr}$ by mc matrix of integer pixel intensities ranging, from 0 to $\mathrm{L}-1, \mathrm{~L}$ is the number of possible intensity values, often 256 .

$$
\begin{aligned}
s_{k} & =T\left(r_{k}\right)=\sum_{j=0}^{k} p_{r}\left(r_{j}\right) \\
& =\sum_{j=0}^{k} \frac{n_{j}}{n} \quad k=0,1,2, \ldots, L-1 .
\end{aligned}
$$

Hence the processed output image is obtained by mapping each pixel with level $r_{k}$ in the input image into corresponding pixel with level $S_{k}$.
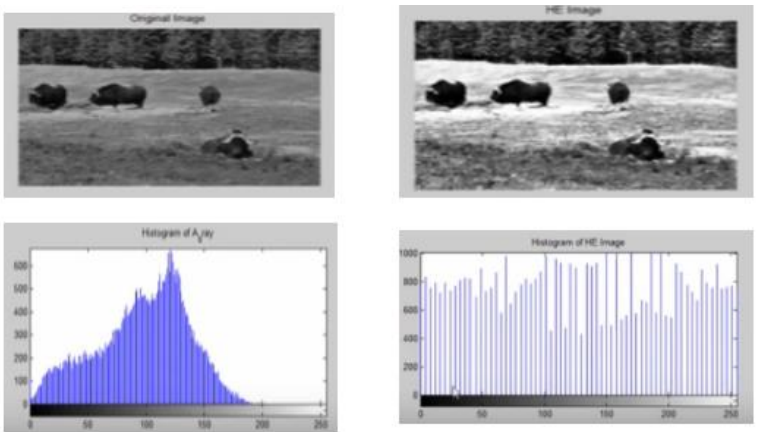

Fig. 5.1.5 Histogram Equalization

Here, the proposed methodology depends on the image processing, we will convert the images as per convenience for change detection using histogram difference. First of all we will get a triggered image as per your desired frame, then generate an RGB image and convert it into a double ( 0 to 1 instead of 0-255) then convert it into grayscale to reduce RGB channel, after this we will generate its histogram and compare it with the real time video for change detection.

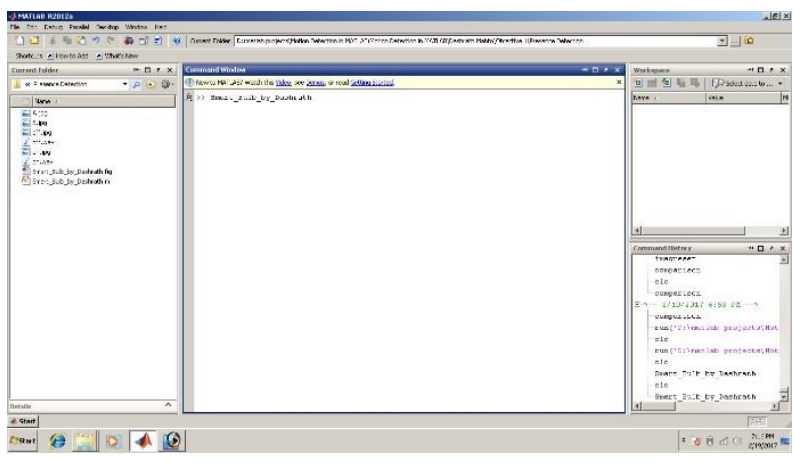

Fig. 5.1.6 Command Window

Fig. 5.1.6 Is a command window from where we run the executing file.

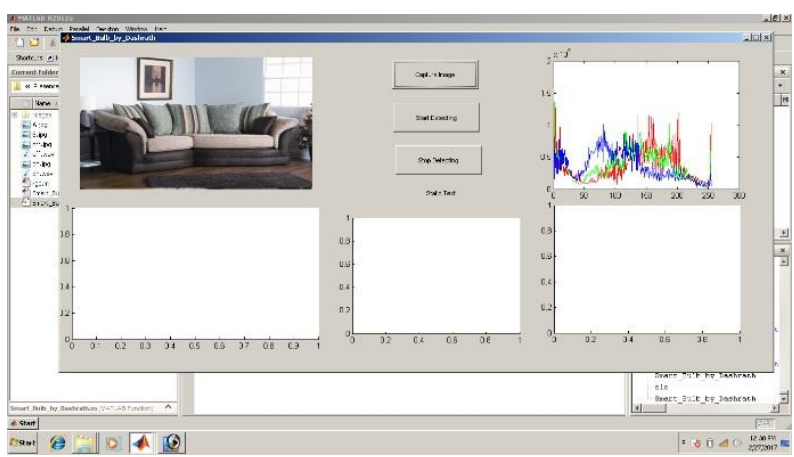

Fig. 5.1.7 Main Window

Fig. 5.1.7 Is a main window where we start executing our main program. So if we calculate the histogram of the background image of a living room as a living room is considered as background image, then the histogram of the vacant living room is very much differs from those real time images where someone is present. So we are detecting these changes in real time and on the basis of these changes we instruct our system. The Histogram is very much sensitive, it means that even a dot in the image will generate different histogram graph, so that is why we compare it on the basis of histogram and we acquire the real time output with high accuracy.

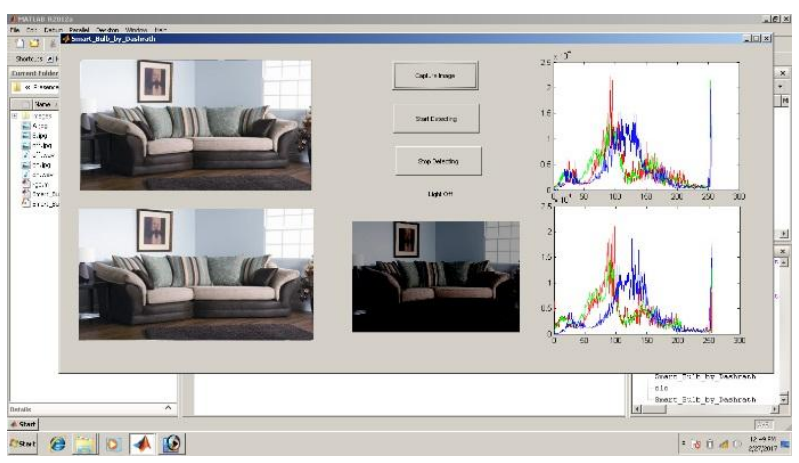

Fig. 5.1.8 No Presence Detected

As we can see there is no one present in the room, so the histogram is same for both the images, so that is why system considered bulb should be turned off because no one is present in the room. 


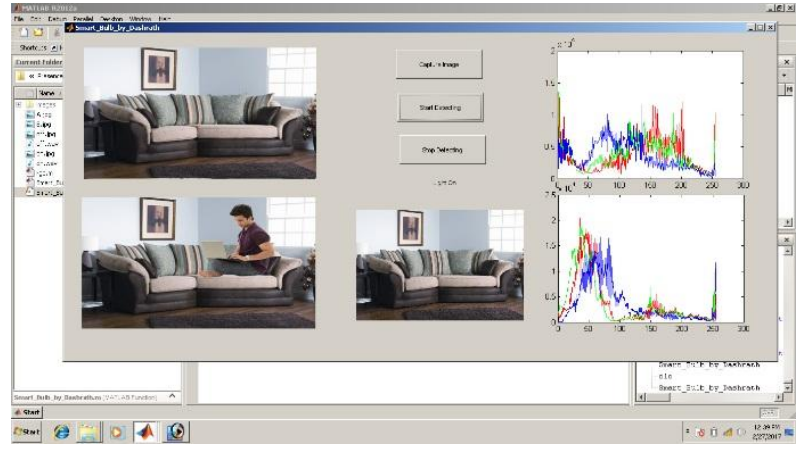

Fig. 5.1.9 Presence Detected

According to the fig 5.1.9 there is someone present in the room, so the histogram of the background image and the source image is very much differ, so light should be remain on because someone is present in the room. Now the histogram for presence detection for all three RGB channels.

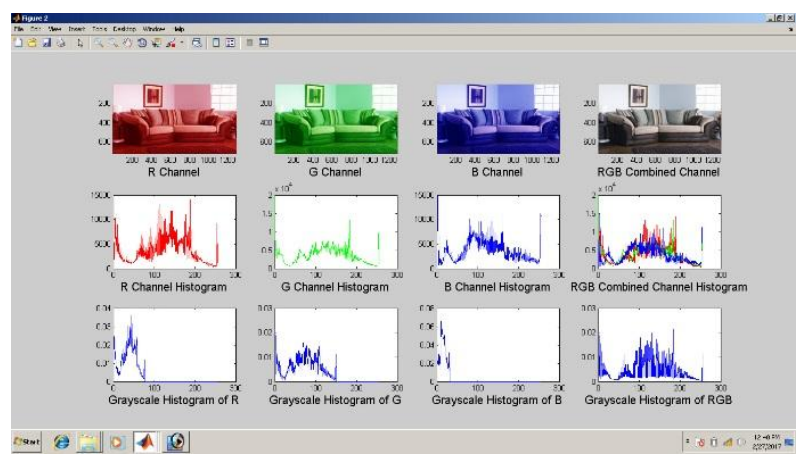

Fig. 5.1.10 Histogram Difference of Separate RGB Channels for $\mathrm{A}$

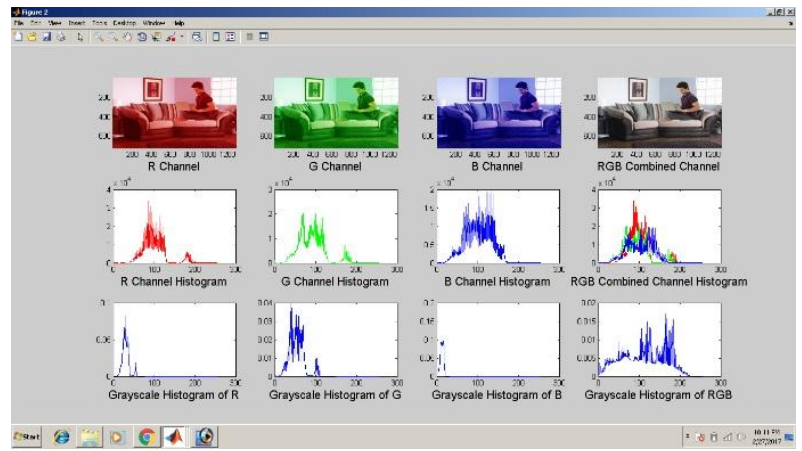

Fig. 5.1.11 Histogram Difference of Separate RGB Channels for $B$

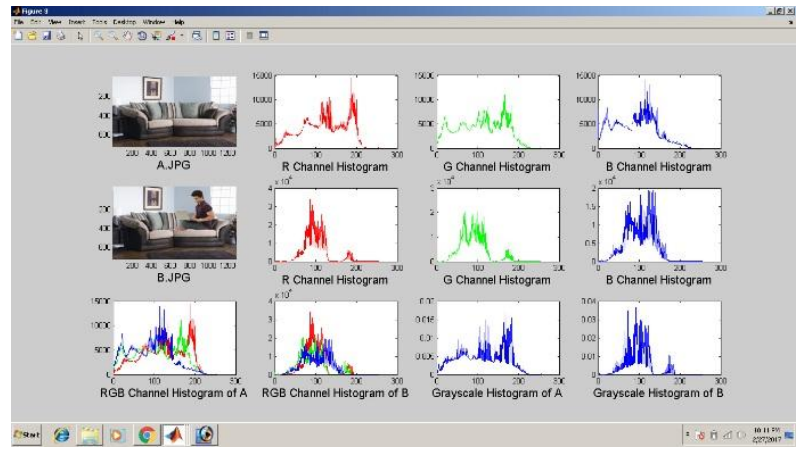

Fig. 5.1.12 Histogram Difference between A and B
Fig 5.1.12 is also a histogram difference between two images in which one image has no presence and another one is the resultant image of source image.

Now the histogram differences for no presence, for separate RGB channels along with grayscale histogram i.e. single channel histogram.

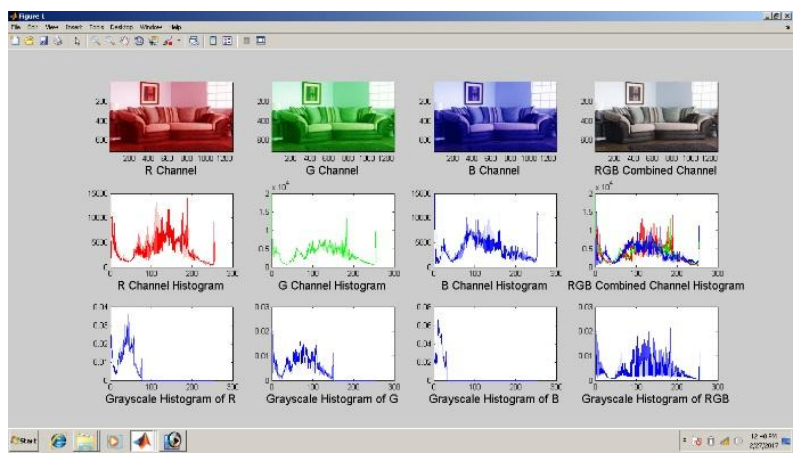

Fig. 5.1.13 Histogram Difference of Separate RGB Channels for $\mathrm{A}$

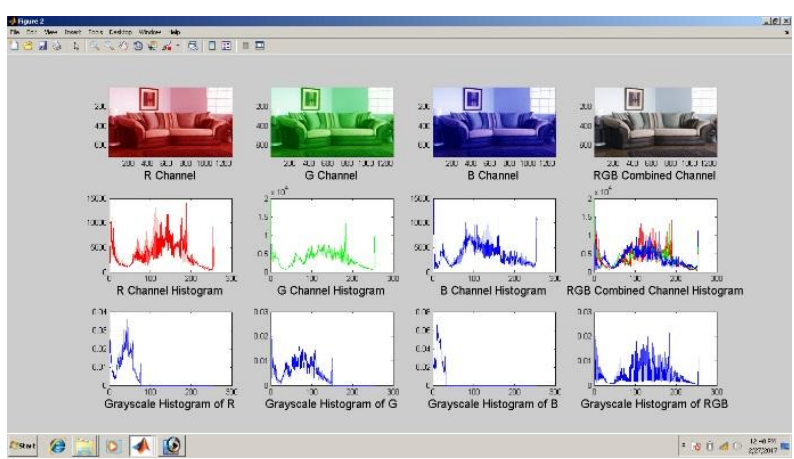

Fig. 5.1.14 Histogram Difference of Separate RGB Channels for B

Fig. 5.1.14 is the histogram of image B for separate channels Red, Green and Blue along with grayscale channel. A is the first image and $\mathrm{B}$ is the second image which have been captured.

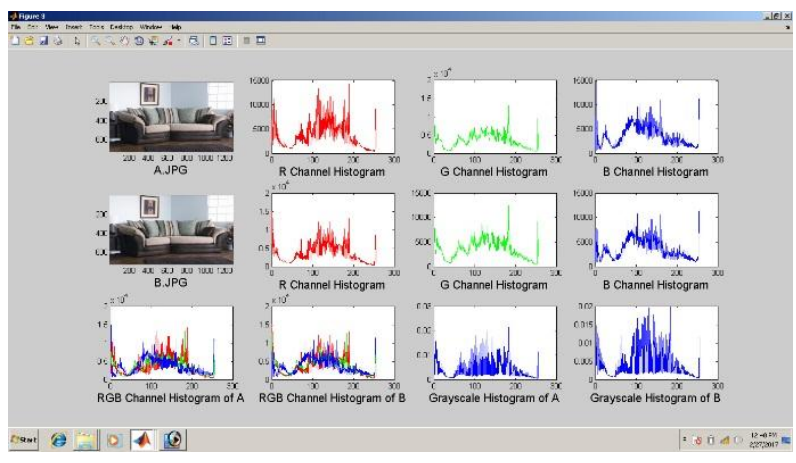

Fig. 5.1.15 Histogram Difference between A and B 


\section{SLEEP DETECTION:}

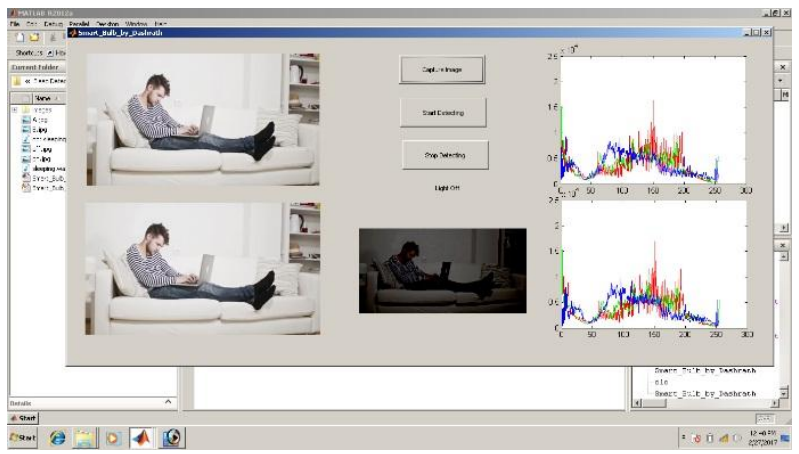

Fig. 5.1.16 Sleep Detection

In fig. 5.1.16 we can see there is a man working and after a while he got sleep, so according to the system bulb should be turned off because you are sleeping and bulb should be turned off.

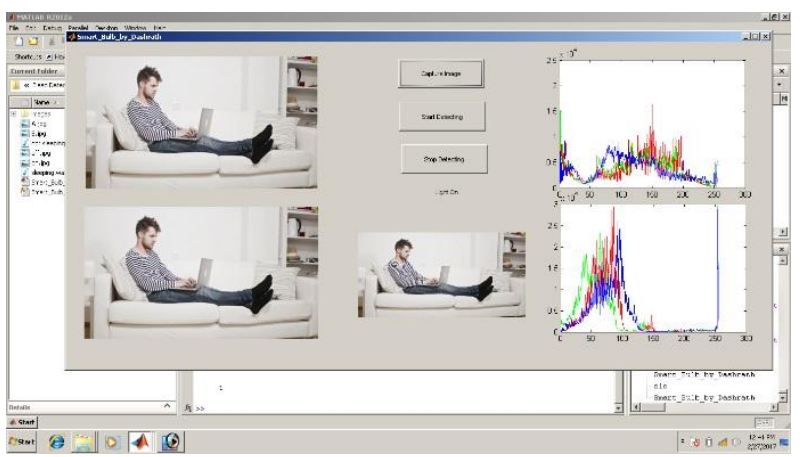

Fig. 5.1.17 No Sleep Detected

According to the fig. 5.1.17, he is not sleeping and light should be remaining turned on which system did. If more than one person is present in the room, then it will check whether all are sleeping or not if all are sleeping, then it will turn off bulb otherwise it will remain turned on.

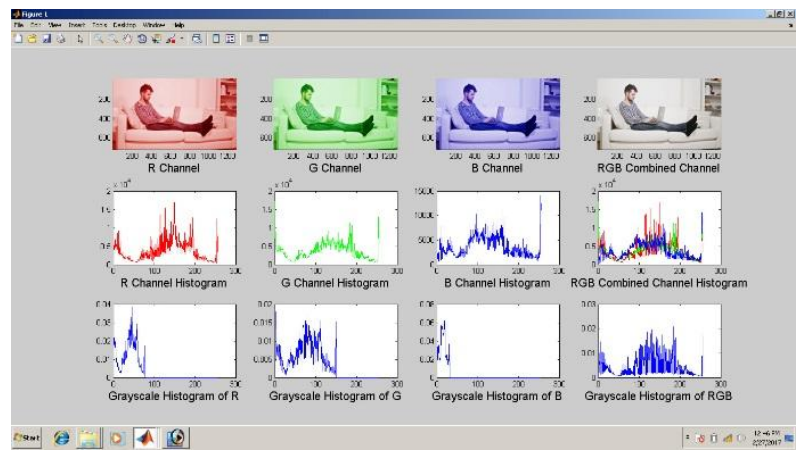

Fig. 5.1.18 Histogram Difference of Separate RGB Channels for $\mathrm{A}$

Fig. 5.1.18 is the histogram of image A where someone is working. Histogram shown for all three channels for RGB.

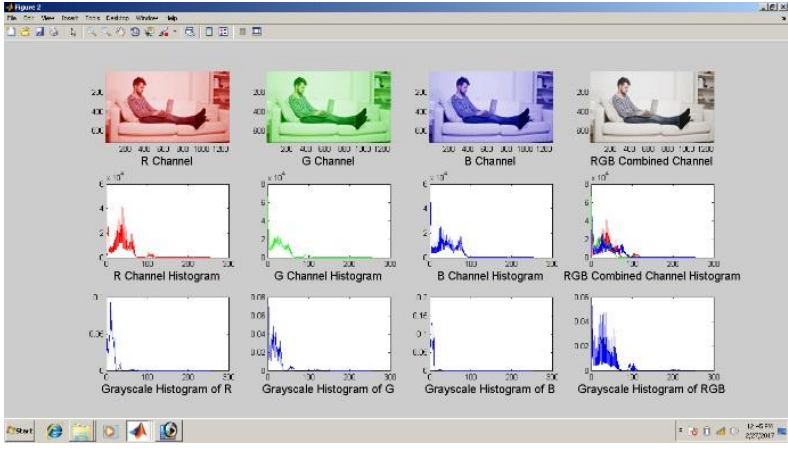

Fig. 5.1.19 Histogram Difference of Separate RGB Channels for $B$

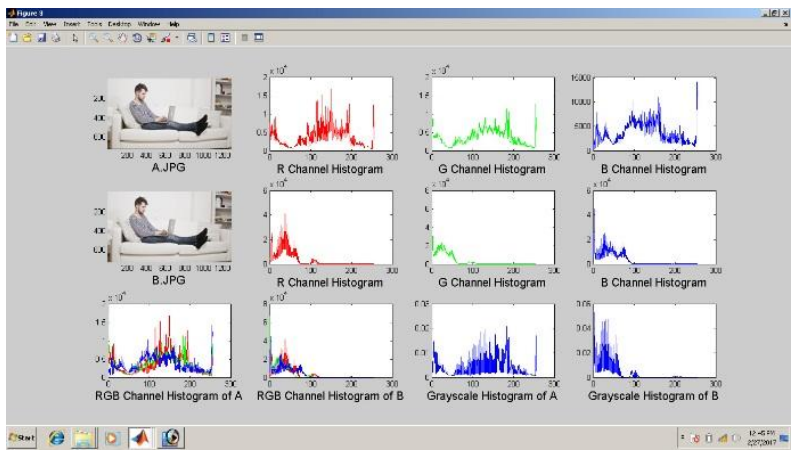

Fig. 5.1.20 Histogram Difference between A and B

Now histogram differences in sleep detected for all three channels RGB, here we also converted the three channels RGB into a single channel for better intensity calculation.

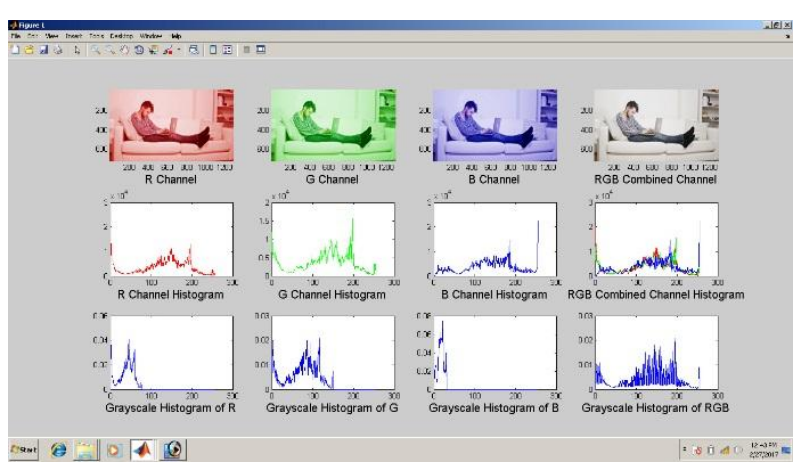

Fig. 5.1.21 Histogram Difference of Separate RGB Channels for $\mathrm{A}$

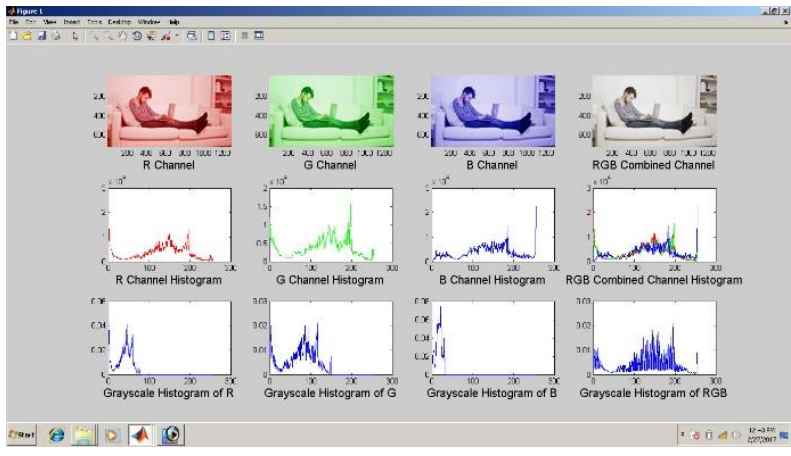

Fig. 5.1.22 Histogram Difference of Separate RGB Channels for B 


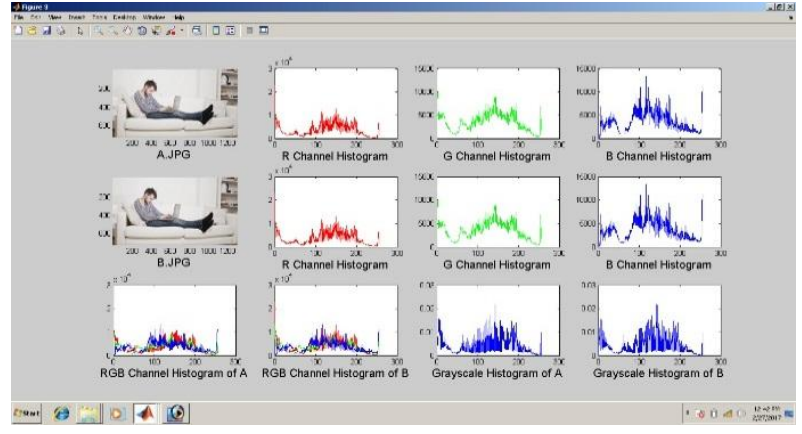

Fig. 5.1.23 Histogram Difference between A and B

We are going to use various approaches of image processing through MATLAB. Let it be more precise by flowchart.

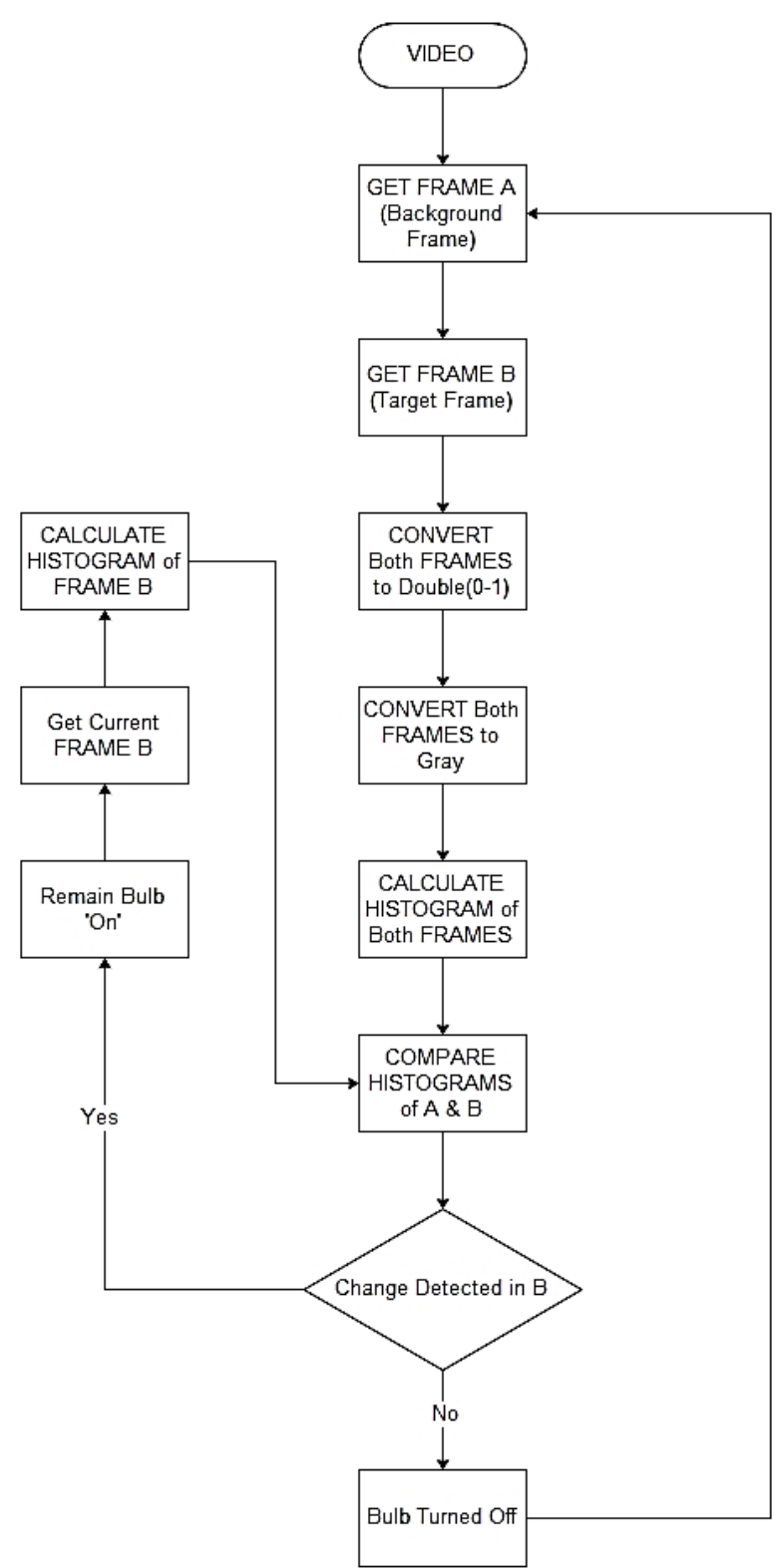

Fig. 5.1.24 Flow Chart of Presence Detection

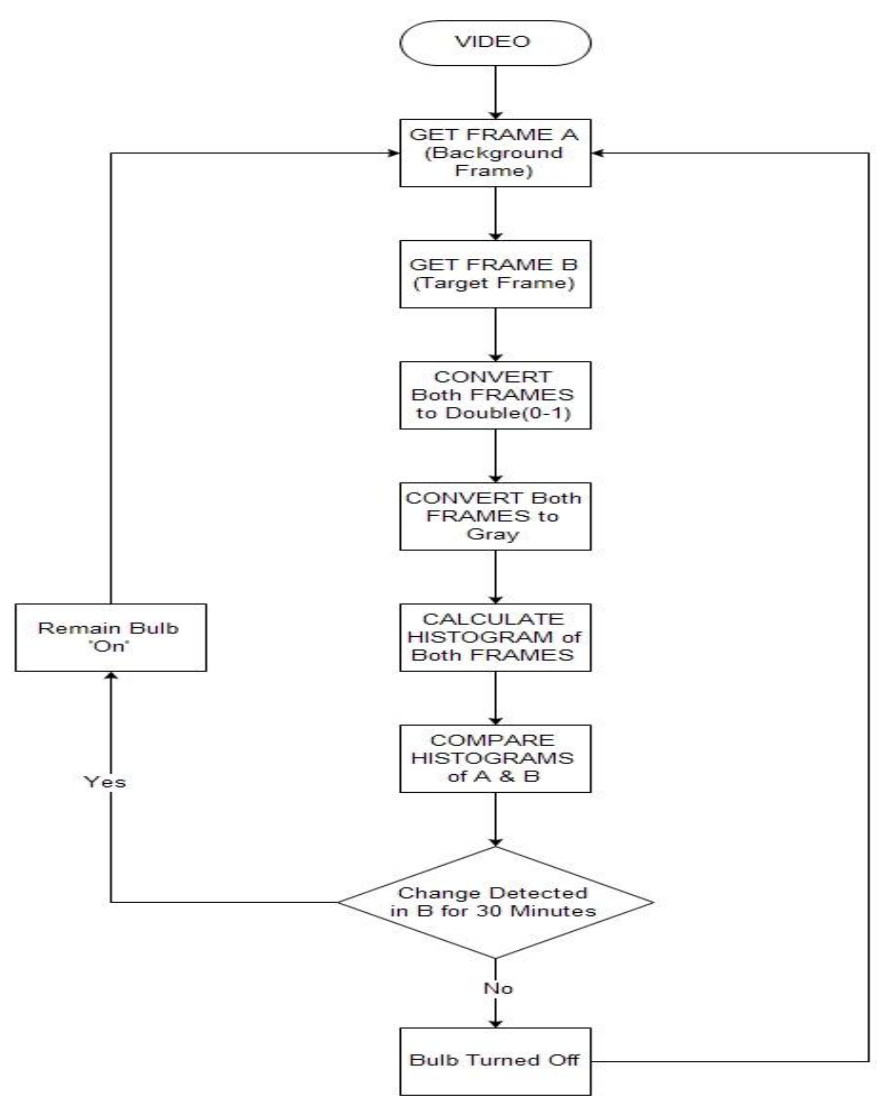

Fig. 5.1.25 Flow Chart of Sleep Detection

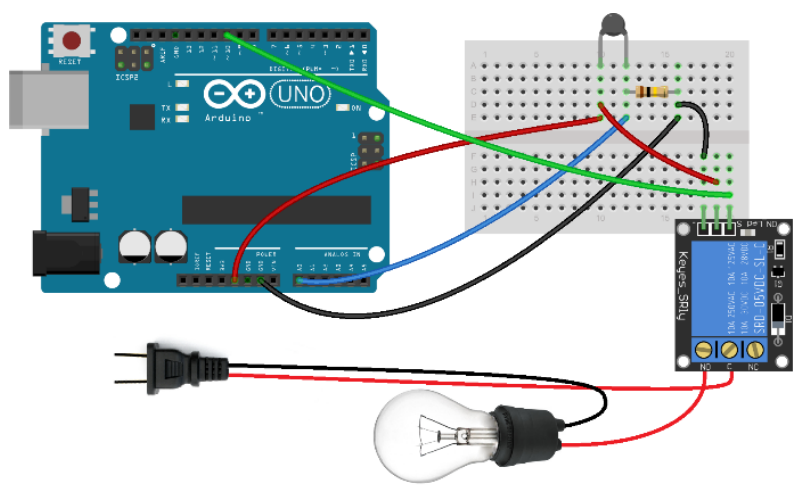

Fig. 5.1.26 Arduino Implementation

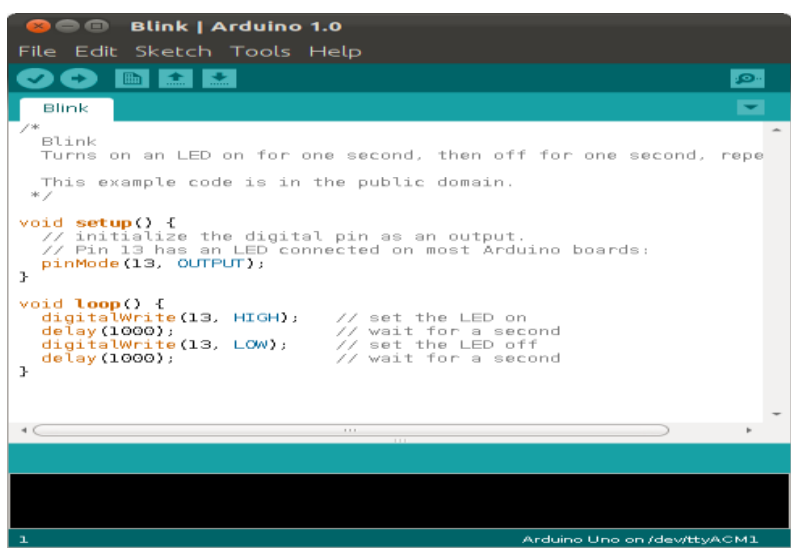

Fig. 5.1.27 Arduino Software 


\section{CONCLUSION}

Thus the real time vision based smart bulb avail you to save your electricity automatically and also save you and your house from electrical casualty. The present system has no potential to work or sense automatically, but the proposed one will definitely avail you an intelligent bulb which turn your life easier as you think. Because we are going to the era of automation where everything will be operated automatically and intelligent enough to work accordingly and known for good decision making.

\section{FUTURE SCOPE}

The current proposed concept of real time vision based smart bulb definitely gets enhanced in future because we can also develop a system which can control your home appliances automatically using an intelligent approach like your fan, cooler, television and many more which will turn you stress less.

\section{REFERENCES}

[1] Daeho Kim, Junghoon Lee and Yeongmin Jang, Jaesang Cha, "Smart LED lighting system implementation using Human tracking US/IR sensor" in IEEE 978-1-4577$1268-5 / 11 / \$ 26.00$ @2011.

[2] Jie Zhang and Hwa Jong Kim, "Design of smart LED lighting switch with learning user's light controlling pattern" in IEEE 978-1-4577-1268-5/11/\$26.00 (02011.

[3] Yen Kheng Tan, Truc Phuong Huynh, and Zizhen Wang, "Smart Personal Sensor Network Control for Energy Saving in DC Grid Powered LED Lighting System" IEEE Tran. on smart grid (c) 2012.

[4] Young Seek Cho, Jaerock Kwon , Seyeong Choi and Dae-Hee Park, "Development of Smart LED Lighting System Using Multi-Sensor Module and Bluetooth Low Energy Technology", IEEE SECON Posters -- IEEE
International Conference on Sensing, Communications and Networking (SECON), 2014.

[5] Zeeshan Kaleem, Ishtiaq Ahmad and Chankil Lee,"Smart and Energy Efficient LED Street Light Control System using ZigBee Network", 12th International Conference on Frontiers of Information Technology in IEEE, 2014.

[6] Sunghoi Park, Soono Seo, Byunghoon Lee, Jinsung Byun, and Sehyun Park, " An Energy Efficient Smart LED Lighting System for Building Energy Management”, IEEE ISCE 2014 1569954593, 2014.

[7] M. Magno, T. Polonelli, L. Benini and E. Popovici,"A low cost, highly scalable Wireless Sensor Network Solution to achieve smart LED light control for Green Buildings", DOI 10.1109/JSEN.2014.2383996, IEEE Sensors Journal, 2015.

[8] Xiaoru Xu, Huiqiang Chen, Budong You, and Xiaobo $\mathrm{Wu}$, "Power Factor Corrected Primary Side Regulated Flyback Controller for Smart LED Lighting System", in IEEE 978-1-5090-0176-7/15/\$31.00 @2015.

[9] Kai-Lun Chen, Hao-Ping Chan, Yu-Cherng Hung, and Shao-Hui Shieh, "A Smart LED Lighting with Multiple Dimming and Temperature Automatic Protection Capabilities", in International Symposium on Computer, Consumer and Control, 2016.

[10] Ivan Chew, Vineetha Kalavally, Chee Pin Tan, and Jussi Parkkinen, "A Spectrally-Tunable Smart LED Lighting System with Closed-Loop Control", DOI 10.1109/JSEN.2016.2542265, IEEE Sensors, 2016.

[11] Qi Yao, Lei Yuan and Yu Bian, "Establishment of Vision Effect Diagram for Optimization of Smart LED Lighting", Published in IEEE Photonics Journal. Volume 8, Number 4, August 2016. 